\title{
Análisis cualitativo y cuantitativo de las materias básicas de base de datos en las memorias de verificación de los títulos universitarios de Grado en Informática en las Universidades Españolas
}

\author{
Contreras J.A., Luengo R., Arias J., Casas L.M. \\ jaconvas@unex.es, rluengo@unex.es,juanaria@unex.es, luisma@unex.es \\ Grupo de Investigación CIBERDIDACT, Departamento de Ingeniería de Sistemas Informáticos y \\ Telemáticos, Centro Universitario de Mérida, Universidad de Extremadura, Mérida, Badajoz-0680o, España
}

DOI: 10.17013/risti.e2.37-53

\begin{abstract}
Resumen - Este documento expone el trabajo realizado sobre el tema de los contenidos básicos de las materias de Base de Datos que pueden formar parte de los títulos de Grado en Informática en las universidades españolas adaptados al EEES1. Se han utilizado dos metodologías de análisis; una primera de análisis cualitativo utilizando el software WebQDA; y otra metodología mediante Redes Asociativas Pathfinder utilizando otro software llamado Goluca. En el software WebQDA se ha tomado como entrada 22 documentos que contienen los contenidos de Base de Datos que aparecen en las memorias de verificación de los estudios de Grado en Informática de universidades españolas. Dicho análisis cualitativo genera una matriz de proximidad de conceptos. Esta matriz sirve como entrada al programa Goluca para realizar un estudio minucioso de la red generada y poder determinar los conceptos nucleares existentes explicados utilizando la teoría de los conceptos nucleares.
\end{abstract}

Palabras-clave: Análisis cualitativo y cuantitativo; base de datos; universidad; Redes Asociativas Pathfinder; Teoría de los "Conceptos Nucleares".

Qualitative and quantitative analysis of basic content of database in verification memoirs of degrees in Computer Science in Spanish Universities

Abstract - This paper presents the work done on the subject of the basic contents
of the materials database that can be part of the bachelor degrees in Computer
Science in the Spanish universities adapted to the EHEA. We used two methods of
analysis; a first qualitative analysis using the software webQDA; and a
methodology through Pathfinder Associative Networks using another software
called Goluca. In webQDA software is taken as input 22 documents containing the

${ }^{1}$ EEES: Espacio Europeo de Educación Superior. (en inglés EHEA). 


\begin{abstract}
database contents to appear in the proceedings of verification Degree in Informatics Spanish universities. This qualitative analysis generates a proximity matrix of concepts. This matrix serves as input to the program Goluca conduct a thorough study of the generated network and determine the key concepts explained using existing theory of nuclear concepts.
\end{abstract}

Keywords: Qualitative and quantitative Analisys; database; university; Pathfinder Associative Networks; Theory of the "Nuclear Concepts".

\title{
1. Introducción
}

Este trabajo resume el proceso realizado en una investigación mixta, cualitativacuantitativa, referida a la extracción y análisis de los conceptos básicos de las materias de Base de Datos en las universidades españolas.

Finalizado el periodo de adaptación de los títulos de Informática a los nuevos Grados en Informática en el EEES, es el momento para extraer información de dichos títulos nuevos y averiguar cuáles son los conceptos básicos sobre Base de Datos que los alumnos deben aprender cuando abordan por primera vez estos estudios.

Teniendo como objetivo fundamental del trabajo la extracción de los conceptos básicos sobre Base de Datos, se utilizaron 22 documentos de diferentes universidades (Contreras J.A., Arias J., Luengo, R. e Hidalgo V., 2013), concretamente las memorias de verificación de títulos en universidades españolas y planes de estudios en universidades extranjeras, que incluían todos los contenidos considerados en ellos básicos.

Toda esta información extraída se analizó, utilizando una metodología cualitativa (Contreras J.A., Luengo R., Arias J. y Castillo A., 2013) a través del software webQDA, es decir, se analizaron los diferentes textos dando como resultado un conjunto de nodos o categorías. Y dado que el número de nodos obtenidos fue bastante numeroso se consideró agruparlos en diez grupos teniendo en cuenta la afinidad temática de los mismos.

Posteriormente se extrajo, mediante la exportación de datos del software WebQDA, la información que se necesitaba para realizar el siguiente análisis cuantitativo en el programa Goluca (Godinho V., 2007). En dicho software, que está basado en la teoría de los Conceptos Nucleares (Luengo R., 2013) creada por el Doctor Casas en su tesis doctoral (Casas L., 2002) en la Universidad de Extremadura, se importó la información anterior y se utilizaron los recursos que éste software proporciona para realizar el análisis cuantitativo; y conseguir así nuestro principal objetivo anteriormente expuesto.

El software Goluca proporciona, por tanto, la discriminación de los conceptos básicos sobre Base de Datos, de entre todos los conceptos que fueron seleccionados inicialmente. Este software permite, a través del uso de las Redes Asociativas Pathfinder (Schvaneveldt R.W., 1990), establecer redes de conceptos en las que se aprecia y cuantifican cada uno de ellos con respecto al conjunto de todos los conceptos. Ello permite discriminar cuales son los conceptos fundamentales de entre todos los conceptos analizados. Estos conceptos serán para nosotros los conceptos nucleares. 
Algunos autores como Fenker (Fenker R.M., 1975), Jonassen (Jonassen D.H., 1990), Preece (Preece P., 1976) y Shavelson (Shavelson R., 1972) están de acuerdo en que procedimientos o técnicas similares a las Redes Asociativas Pathfinder, como Análisis de Componentes Principales, Análisis de Cluster o Escalamiento Multidimensional, sirven para definir las estructuras cognitivas.

\section{Fundamentos. La teoría de los Conceptos Nucleares}

Para los doctores Casas y Luengo, los creadores de la Teoría de los Conceptos Nucleares, en adelante TCN, esta teoría es una "propuesta de integración". La razón es que tiene su fundamento en otras anteriores, particularmente las de (Piaget J., 1978) o (Ausubel D.P., Novak J.D. y Hanesian H., 1978), basándose en muchas de sus ideas, y recogiendo aportaciones de otros campos y de los resultados de investigación.

Por lo que se refiere a las teorías de Piaget, los autores creen que "su aportación permite un análisis muy detallado de los fenómenos que influyen en adquisición del conocimiento, como por ejemplo el de la acomodación, que Piaget utiliza, pero que no describe en profundidad. En cuanto a las de Ausubel, la TCN propone modificaciones, basándose en datos experimentales, de algunos de sus presupuestas, pero su propuesta se basa en ellas y las integra aprovechando los datos experimentales y las aportaciones de otras Ciencias".

Los elementos fundamentales del modelo de la TCN, se esquematizan en la Figura 1. En la Figura 2 vemos cuales son las herramientas o técnicas con la que cuenta cada una de las teorías para el desarrollo de dicha teoría. Así, para la Teoría de los Conceptos Nucleares disponemos de al menos dos herramientas básicas como son las Redes Asociativas Pathfinder y el programa Goluca.

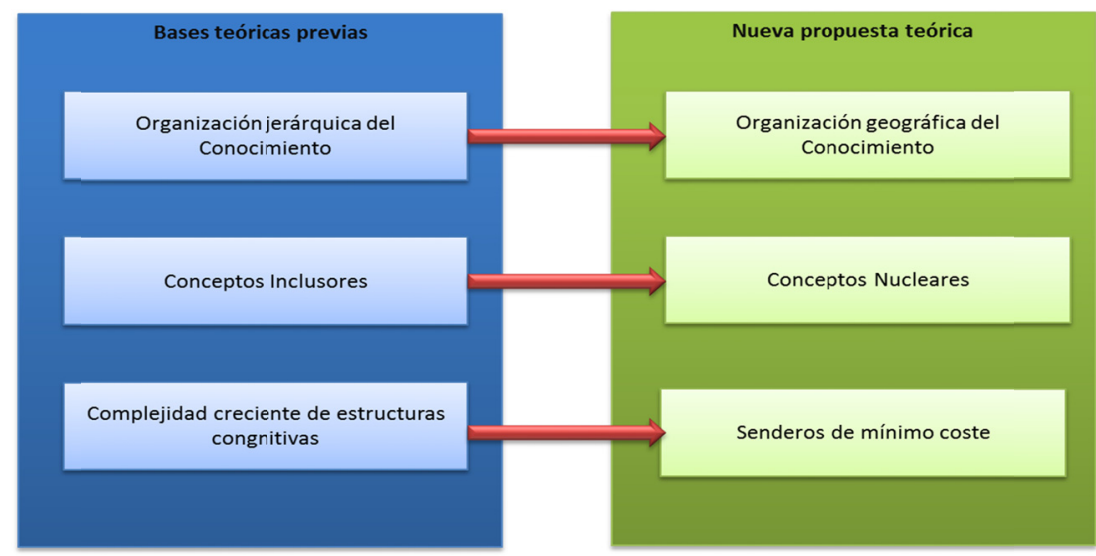

Figura 1 - Elementos de la Teoría de los Conceptos Nucleares (TCN). 


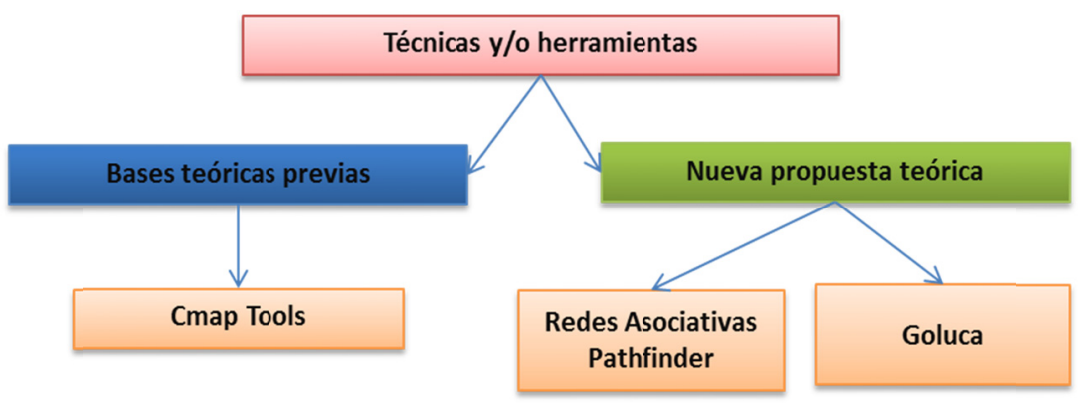

Figura 2 - Herramientas asociadas con las distintas teorías.

Los puntos en los que difieren con las ideas de Piaget, Ausubel y Novak proponiendo alternativas nuevas (Luengo R., Casas L., Mendoza M. y Arias J., 2011) los recogemos en los siguientes apartados.

\subsection{Organización geográfica del conocimiento}

Según describen los autores en (Casas L., 2002), (Casas L. y Luengo R., 2003b), (Casas L. y Luengo R., 2004a), (Casas L. y Luengo R., 2004b), (Casas L. y Luengo R., 2005) y en (Casa L. y Luengo R., 2013), su nueva propuesta teórica parte de una idea muy sencilla, la adquisición del conocimiento y su almacenamiento en la estructura cognitiva, en términos generales, sigue un proceso análogo a la adquisición del conocimiento del entorno físico, y nos da un ejemplo: así como un cartógrafo elabora un mapa geométrico, las personas elaboran sus mapas cognitivos sobre entornos físicos o sus conocimientos en un área.

También describen que si analizamos la adquisición del conocimiento de nuestro entorno, como por ejemplo el de nuestra ciudad, este se puede producir en tres etapas:

- Por la adquisición de ciertos "hitos" sobresalientes del terreno, como edificios singulares o paisajes característicos (conocimiento de hitos).

- La adquisición del conocimiento de una ruta, que es la capacidad de navegar en un hito a otro, sin considerar las áreas de alrededor (conocimientos de rutas).

- Por último el mapa cognitivo del entorno físico no estaría completo sin una visión de conjunto, es decir, conocer todas las conexiones entre los hitos a través de las rutas (conocimientos de conjunto).

Como vemos la primera contribución que hace esta nueva teoría se refiere a como el individuo organiza su conocimiento dentro de la estructura cognitiva, en una forma geométrica, es decir, como un mapa de una ciudad donde no hay elementos jerárquicos, sino elementos relevantes y centrales.

Lo cual viene a contradecir lo que Ausubel menciona sobre este tema y el aprendizaje significativo, quien describe que los conocimientos se adquieren bajo estructuras jerárquicas (de lo general a lo especifico) y no geométrica como lo mencionan los autores en su nueva teoría. 


\subsection{Conceptos nucleares}

Los autores coinciden con la idea desarrollada en sus trabajos por Ausubel, Novak y Hanesian (Ausubel D.P., Novak J.D. y Hanesian H., 1978), en que el conocimiento se construye sobre la base de lo que previamente se conoce, idea que responde al enfoque general del constructivismo, pero difieren en la consideración acerca de la forma en que tiene lugar este proceso, y ello en vista de los resultados experimentales que se mostraran más adelante.

La teoría de estos autores propone que hay ideas de nivel superior, llamadas inclusores que sirven como anclaje para otras. A estas ideas es a las que se refieren al afirmar que es necesario para lograr un mejor aprendizaje y la retención del material lógicamente significativo y nuevo la disponibilidad dentro la estructura cognoscitiva de ideas de afianzamiento específicamente pertinentes a un nivel de inclusividad adecuado.

A partir de esta noción, se entiende que la construcción del aprendizaje es claramente jerárquica y el tipo de aprendizaje superior es el aprendizaje subordinado, en el que las nuevas ideas son relacionadas de forma subordinada con las ideas previas, que son de mayor nivel de abstracción, generalidad e inclusividad.

Según este planteamiento experimental, si la existencia de los inclusores tal como los entienden Ausubel, Novak y Hanesian (Ausubel D.P., Novak J.D. y Hanesian H., 1978), se confirmara, en la estructura cognitiva del alumno debieran aparecer como más destacadas estas ideas, de nivel superior. Sin embargo, esto no coincide con los datos obtenidos en su experimentación.

Frente a la concepción jerárquica del conocimiento, conciben más bien, como hemos explicado antes, una concepción "geográfica". Cuando conocemos un territorio nuevo, no aprendemos el país, luego la región y luego el pueblo donde estamos, correspondiendo país, región y pueblo a conceptos sucesivamente más inclusivos. De forma diferente, y como se ha propuesto antes, se aprenden unas marcas en el territorio, unas referencias geográficas, que no tienen que ser las más generales, sino que quizá sean una ciudad o un edificio concretos, y luego vamos ampliando nuestro conocimiento del territorio añadiendo nuevos hitos. Remarcan esta idea porque les parece determinante: el aprendizaje no tiene por qué producirse en estadios de mayor a menor inclusividad, y creen que no es esa la forma general del aprendizaje. Posiblemente eso ocurra con los alumnos y sus conocimientos de ciertas cosas: existe un conocimiento parcial, fragmentario, y a lo largo de la escolaridad lo van construyendo y refinando. El aprendizaje, en este sentido, y como indicábamos en páginas anteriores, es un proceso de ajuste de las representaciones mentales del alumno.

Pero este proceso de ajuste no supone una reestructuración total de los conocimientos anteriores, sino que siempre se construye a partir de las estructuras previas.

Según esta teoría, los conocimientos no se van organizando a partir de conceptos más inclusivos a otros más sencillos. Esto quizá ocurra al final, cuando se tiene una visión de conjunto, pero no al principio del conocimiento. Se produce, tal como hemos indicado, por un sistema de "acrecentamiento", tal como el señalado por Rumelhart (Rumelhart D.E., 1980), primero hitos del paisaje, después rutas y después visión general del mapa. 
También según esta concepción, y dado que no consideran que el aprendizaje se apoye siempre en una estructura jerárquica, no tiene por qué haber conceptos ni más importantes ni de menor nivel, sino que hay simplemente conceptos que sirven como anclaje la estructura cognitiva del alumno. La cuestión clave para la práctica educativa es que quizás, en el proceso de enseñanza, el profesor no sepa cuáles son las ideas más generales en la estructura cognitiva del alumno, y pudiera estarle presentando algo que no es significativo para él. El mismo Novak, al hablar sobre el papel de los organizadores previos y su construcción, manifestaba que su elección dependía de cuáles eran los inclusores relevantes no sólo para los materiales de aprendizaje que iban a presentarse, sino para la población a la que se dirigía. Frente a ello, parece consistente pensar que lo más interesante sería identificar cuáles son los "hitos" en el territorio de conocimiento en que se mueve el alumno, sus "conceptos nucleares".

\subsection{Senderos de mínimo coste}

La consideración jerárquica del conocimiento parece tener como consecuencia lógica la adquisición de una mayor complejidad en la estructura cognitiva conforme aumenta la cantidad de conceptos y las relaciones entre ellos, que va produciéndose cuando se adquieren nuevos aprendizajes. Sin embargo, al analizar, los autores, los datos experimentales (Casas L., 2002), mientras mayor es la edad de los alumnos y más avanza su aprendizaje, más simples aparecen las representaciones de las relaciones entre conceptos que se obtienen con las Redes Asociativas Pathfinder.

Se puede interpretar este hecho considerando que, a pesar de que en la estructura cognitiva del alumno aparecen cada vez más elementos y más relaciones entre ellos, se utilizan subestructuras cada vez más simples. Loa autores creen que en una situación dada que requiera utilizar los aprendizajes adquiridos y almacenados en la estructura cognitiva, en lugar de recurrir a las relaciones entre todos los conceptos presentes, en una estructura compleja, se recurre a las relaciones más simples, pero que resultan más significativas, a lo que se denomina "senderos de mínimo coste". La elección de este nombre es intencionada, pues responde, por una parte, a la propia lógica de la representación gráfica que veremos al hacer uso de las Redes Asociativas Pathfinder, y por otra, a las aportaciones de la Biología a la Ciencia Cognitiva (Edelman G., 1992). Efectivamente, ante un nuevo aprendizaje, la activación de un mapa neuronal supone también la activación de un circuito que integran otros que están asociados a él, pero sólo de algunos, no de toda la estructura cerebral completa, pues esto sería muy costoso en términos energéticos. Esto significa que en cada momento, al hacer uso de un aprendizaje, el sujeto sólo activa los mapas neuronales que, por un proceso previo de selección por la experiencia han resultado reforzados frente a otros que han desaparecido. Como ocurre en otros aspectos vitales, la estructura cognitiva funciona por un principio de mínima energía.

A nivel psicológico, la elección de unos circuitos de conexiones u otros, o lo que es lo mismo, de distintos senderos, depende también de un proceso de selección de tipo probabilístico, el cual en función de las experiencias previas, nos aconseja elegir un enlace u otro cuando se dispone de distintas alternativas. Se escoge aquel sendero que tiene más posibilidades de éxito con menor coste. 
La metáfora geográfica que se viene utilizando permite también entender mejor el concepto de "senderos de mínimo coste". Las personas en cada tipo de viaje que emprenden, y para cada intención, utilizan un mapa distinto: no es necesario el mismo mapa si se quiere hacer turismo y visitar localidades pintorescas, que si el viaje es de trabajo y se necesita ahorrar tiempo en el desplazamiento. Efectivamente, con la edad y el conocimiento, las redes cognitivas se hacen más complejas. Este es un razonable principio de la teoría del desarrollo intelectual, pero también es razonable pensar en los términos que hemos expuesto. No son principios contradictorios, sino complementarios.

Continuando con la metáfora geográfica, cuando llegamos a vivir a un nuevo país, tenemos un conocimiento pobre de su red de carreteras, aunque cuando pasan los años cada vez la conocemos mejor. Y cuando tenemos un buen conocimiento, utilizamos siempre las mejores carreteras para ir a nuestro trabajo, aunque a veces, por motivos turísticos, podamos ir por carreteras secundarias. Pero habitualmente, preferimos ciertos enlaces y dejamos de utilizar otros. Eso no quiere decir que nuestro conocimiento de la red de carreteras sea peor, sino precisamente lo contrario: lo utilizamos de la forma más simple, pero más efectiva. De este modo, cuando se requiere resolver una tarea matemática, sea éste del tipo que sea, se seleccionan los conceptos clave relacionados con él, así como los detalles relevantes para el caso y los procedimientos usualmente aplicables para su resolución. Esta información se encuentra almacenada en la estructura cognitiva en forma de esquemas mentales. Pero sólo se activan y se relacionan entre sí aquellos esquemas que son relevantes en la situación concreta de que se trate, y no otros, que resultan descartados, por un proceso de selección que está determinado en gran parte por las experiencias previas. La conexión más efectiva entre conceptos clave, detalles y procesos de resolución, forman lo que antes hemos denominado un sendero de mínimo coste.

La capacidad de seleccionar cuáles son los senderos de mínimo coste es una característica del alumno que resuelve eficazmente los problemas, pero del mismo modo, la capacidad de escoger otros senderos, la flexibilidad para recorrer caminos no usuales, es lo que determina al alumno creativo. Tanto en uno como en otro caso la elección se rige por el mismo principio: la evaluación del coste y de la probabilidad de éxito, determinadas ambas, por las experiencias previas.

En la figura 3 se representa de forma esquemática, los distintos senderos numerados del 1 al 5 , que se pueden seguir para la resolución de un problema. La conexión más efectiva entre conceptos clave, detalles y procesos de resolución forman por tanto "un sendero de mínimo coste".

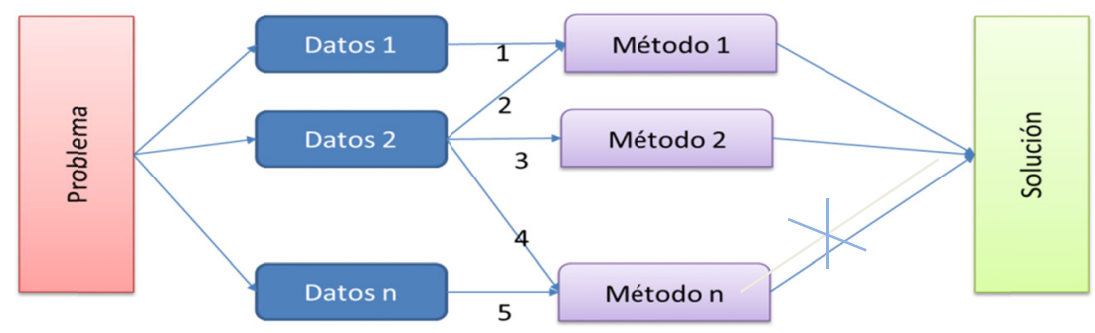

Figura 3 - Distintos "senderos" en la resolución de un problema. 
Con independencia del hecho de que en algunos de ellos (4 y 5) no conducen a la solución, por elegir los datos o los métodos inadecuados, queremos llamar la atención sobre la mayor longitud, el mayor coste, de unos frente a otros (Casas L., 2002).

\section{Metodología.}

Los pasos que se han seguido para llevar a efecto el trabajo global han sido los siguientes pasos:

\subsection{Introducción de los datos en el software WebQDA.}

En un trabajo nuestro anterior (Contreras J.A., Arias J., Luengo, R. e Hidalgo V., 2013) realizamos un proceso minucioso de análisis de los textos de las memorias de verificación de los títulos de Grado en Informática de distintas universidades. Por cada texto de cada memoria generamos un fichero en formato Word de Microsoft y lo incorporamos como fuente interna a nuestro proyecto WebQDA. Podemos apreciar un ejemplo de la lista de las fuentes obtenidas en la siguiente figura 4:

\begin{tabular}{|c|c|c|c|c|c|c|c|c|c|c|c|}
\hline \multicolumn{11}{|c|}{ Archivo Edotar Ver Hiștorial Marcadores Herranientas Ayuda } & \\
\hline \multicolumn{8}{|c|}{ 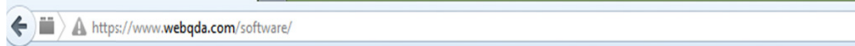 } & \multicolumn{4}{|c|}{$\hat{A} \nabla C$ B-Google } \\
\hline \multicolumn{12}{|c|}{ 3. Más visitados $\mathrm{m}$ Comenzar a usar Firefox } \\
\hline & \multicolumn{2}{|c|}{ Administroion } & \multicolumn{2}{|l|}{ Codfraoün } & Clasiflicatin & \multicolumn{2}{|c|}{ Consulis } & \multicolumn{2}{|c|}{ 证 cidtf } & \multicolumn{2}{|c|}{ Español } \\
\hline WEBपDA & \multicolumn{2}{|r|}{ Aevalzart Nuevo texto Nuevo video } & \multicolumn{2}{|c|}{$\sum_{\text {Eirnar noonicar }}$} & $\begin{array}{l}\text { Copiar } \\
\text { Corter } \\
\text { Pegger }\end{array}$ & \multicolumn{2}{|c|}{ 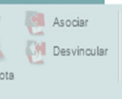 } & \multicolumn{2}{|c|}{ Nueva carpets } & & \\
\hline \multicolumn{12}{|l|}{ Q Fuentes } \\
\hline$\triangleright$ Fuentes inte'nas & $C e$ & es_andalucia_almeria & & & Texto & 11 & 11 & 17-07-2013 17:57:03 & ja & & 17 . \\
\hline$D$ Fuentes externas & 8 & es_aragon_zaragoza & & & Texto & 10 & 10 & 17-07-2013 17:57:34 & $\mathrm{ja}$ & & 17 . \\
\hline \multirow[t]{10}{*}{$\gg$ Notas } & 8 & es_asturias_oviedo & & & Texto & 14 & 14 & 17-07-2013 17:57:47 & ja & & 17 . \\
\hline & 8 & es_baleares_islasbaleares & & & Texto & 8 & 8 & 17-07-2013 177:57:58 & ja & & 17 . \\
\hline & 8 e & es_cararias__aspalmas & & & Texto & 14 & 14 & 17-07-2013 17:58:13 & ja & & 17 . \\
\hline & 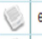 & es_cartabria_cantabria & & & Texto & 19 & 21 & 17-07-2013 17:58:33 & ja & & 17 . \\
\hline & 8 & es_castillalamancha_castillalamancha & & & Texto & 27 & 31 & 17-07-2013 17:58:44 & $\mathrm{ja}$ & & 17 . \\
\hline & Q & es_castillayleon_ourgos & & & Texto & 7 & 7 & 17-07-2013 17:58:54 & $\mathrm{ja}$ & & 17. \\
\hline & 8 & es_cataluña_barcelona & & & Texto & 12 & 12 & 17-07-2013 177:59:12 & ja & & 17 . \\
\hline & 8 & es_extemadura_merida & & & Texto & 10 & 10 & 17-07-2013 177:59:22 & ja & & 17. \\
\hline & 8 & es_galcia_vigo & & & Texto & 24 & 25 & 17-07-2013 177:59:31 & $\mathrm{ja}$ & & 17. \\
\hline & e & es_madrid_politecnica & & & Texto & 12 & 14 & 17-07-2013 177.59:56 & ja & & 17. \\
\hline O Fuentes & 8 & es_national_uned & & & Texto & 28 & 29 & 17-07-2013 18:00:18 & ja & & 17. \\
\hline 4) Codificación & C & es_nalarra_publicadenavarra & & & Texto & 37 & 41 & 17-07-2013 18:00.27 & $\mathrm{ja}$ & & 17. \\
\hline il Consulta & 8 & es_paisvasco_guipuzcoa & & & Texto & 22 & 23 & 17-07-2013 18:00:45 & ja & & 17. \\
\hline \multirow{4}{*}{ Fontes Internas } & 8 & es_valencia_valencia & & & Texto & 60 & 65 & 17-07-2013 18:00:57 & ja & & 17 . \\
\hline & 8 & eeuu_berkeley_ieor115 & & & Texto & 10 & 10 & 17-07-2013 18:01:56 & ja & & 17 . \\
\hline & C & eeuu_mit_6893 & & & Texto & 14 & 14 & 17-07-2013 18:02:09 & ja & & 17. \\
\hline & i & inglateta__imperial_130 & & & Texto & 10 & 12 & 17-07-2013 18:02:19 & ja & & 17 . \\
\hline
\end{tabular}

Figura 4 - Listado de las fuentes en weqbQDA.

Podemos observar en la siguiente figura 5, de forma más amplia que la figura anterior, un contenido típico de los textos fuentes que han sido utilizados en el análisis cualitativo a través de webQDA. 
1.2. Editar Texto

\begin{abstract}
TEMA 1: Sistemas de Gestión de Bases de Datos. SubTema 1.1: 1.1 Origen y evolución de las Bases de Datos. SubTema 1.2: 1.2 Concepto de Base de Datos: Objetivos de las Bases de Datos. SubTema 1.3: 1.3 Independencia de Datos. Arquitectura de Bases de Datos. SubTema 1.4: 1.4 Sistemas de Gestión de Bases de Datos. SubTema 1.5: 1.5 Administración de Bases de Datos. TElMA2: Introducción al Diseño de Bases de Datos. SubTema 2.1: 2.1 El Problema del Diseño de una Bases de Datos. SubTema 2.2: 2.2 Etapas del Diseño. SubTema 2.3: 2.3 El Modelo de Datos Entidad-Relación Extendido. TEMA3: Fundamentos del Modelo Relacional. SubTema 3.1: 3.1 Estructuras de las Bases de Datos Relacionales. SubTema 3.2: 3.2 Restricciones de Integridad. SubTema 3.3:3.3 Teoria de las Dependencias. SubTema 3.4: 3.4 Lenguajes Relacionales. Álgebra Relacional. TEMA4: Diseño en el Modelo Relacional. SubTema 4.1: 4.1 Introducción. SubTema 4.2: 4.2 Normalización por medio de Dependencias Funcionales. SubTema 4.3: 4.3 Diseño Lógico. Del Modelo ERR al Ilodelo Relacional. TEMA5: Introducción a las Bases de Datos Activas. SubTema 5.1: 5.1 Conceptos Básicos. SubTema 5.2: 5.2 Reglas de Comportamiento. SubTema 5.3: 5.3 Disparadores. Aplicadiones.
\end{abstract}

$\mathrm{FB} 4, \mathrm{CC} 12, \mathrm{CC} 13$

Figura 5 - Ejemplo de texto recogido en las fuentes webQDA.

\title{
3.2 Generación y jerarquización de los nodos en el software webQDA
}

El siguiente paso que realizamos fue crear la estructura de los nodos (Contreras J.A., Luengo R., Arias J. y Castillo A., 2013), es decir, la categorización y asignación de las distintas partes de los textos a un nombre o cualidad. De tal forma que algunas partes de textos en distintas fuentes, que hicieran referencia a un mismo concepto o cualidad, se llamasen de la misma forma. En la mayoría de los casos optamos por llamarlos con una frase y no por una única palabra, y también optamos por que esta frase pudiera corresponder con una frase contenida en alguno de los textos a ser posible. Esta asignación de nombres se fue realizando en el mismo momento en el que se realizó el análisis cualitativo. Como resultado obtuvimos un conjunto de nodos bastante numeroso, por lo que posteriormente se crearon, a nivel superior, grupos que contuvieran un conjunto de nodos que estuvieran relacionados temáticamente. Se estableció así, una relación de nodos con una estructura jerárquica. Podemos apreciar en la figura 6 un ejemplo de los nodos con su estructura jerarquizada:

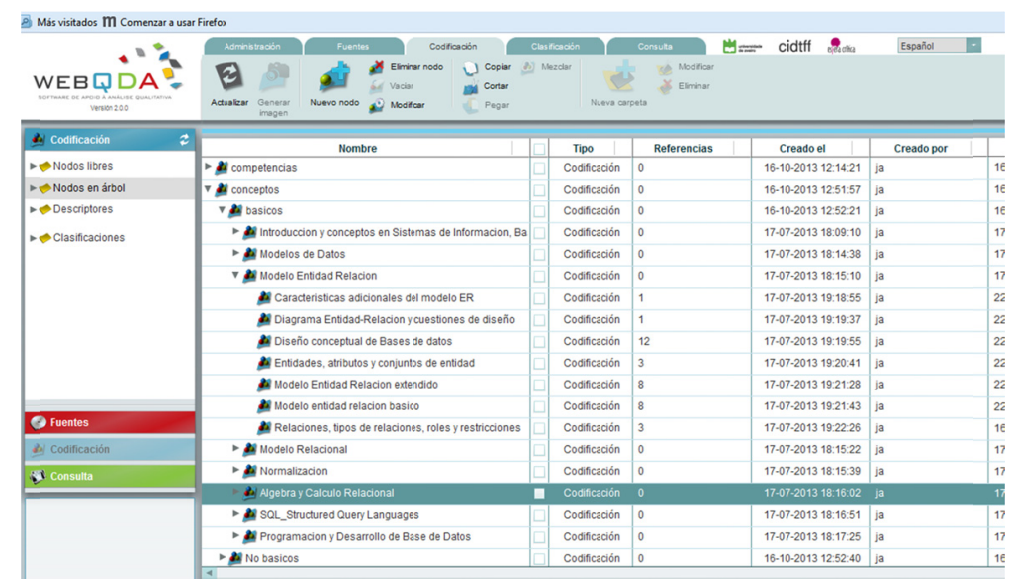

Figura 6 - Listado de nodos con sus jerarquías en webQDA. 


\subsection{Realización del Análisis Cualitativo en el software WebQDA}

Como ya hemos puesto de manifiesto en anteriores trabajos realizados (Contreras J.A., Luengo R., Arias J. y Castillo A., 2013), se procedió a realizar el trabajo de campo de análisis cualitativo manejando todas las características que proporciona el software webQDA, obteniendo como resultado el proceso de categorización de todos los textos fuentes que disponíamos para realizar dicho análisis.

\subsection{Generación y exportación de las matrices en el software WebQDA}

Este software, gracias a los acuerdos llevados a cabo entre la empresa diseñadora del mismo y el grupo de investigación CIBERDIDACT, permite obtener un tipo de consulta, dentro de las consultas de matrices, llamada matrices triangulares.

Esta consulta recoge un tipo de resultado del análisis en el que pone de manifiesto que en cada casilla de la matriz (valor donde coincide una fila con una columna) tenemos el número de veces que han aparecido juntos dos nodos (categorías o conceptos) en todas las fuentes utilizadas para el análisis. Con ello podemos intuir que a mayor valor en una casilla dicho par de nodos están más relacionados que otro par de nodos que tenga menor valor; esto sucede porque hay coincidencia de citación en diferentes textos. Dicha matriz es una matriz simétrica, por lo que sólo es necesario tener información de una de las partes de la matriz. El valor de la diagonal principal es el valor de las veces que ha sido citado un nodo consigo mismo, en este caso el valor de la diagonal principal de la matriz es el número de fuentes en las que ha sido citado dicho nodo. Podemos ver un ejemplo de esta matriz obtenida por el programa en la siguiente figura 7 , donde aparece el nombre del grupo "Álgebra y Cálculo relacional" que tiene seis nodos: álgebra y cálculo relacional, cálculo relacional orientado a dominios, cálculo relacional orientado a tuplas, lenguajes relacionales, operaciones básicas del álgebra relacional y operaciones relacionales adicionales.

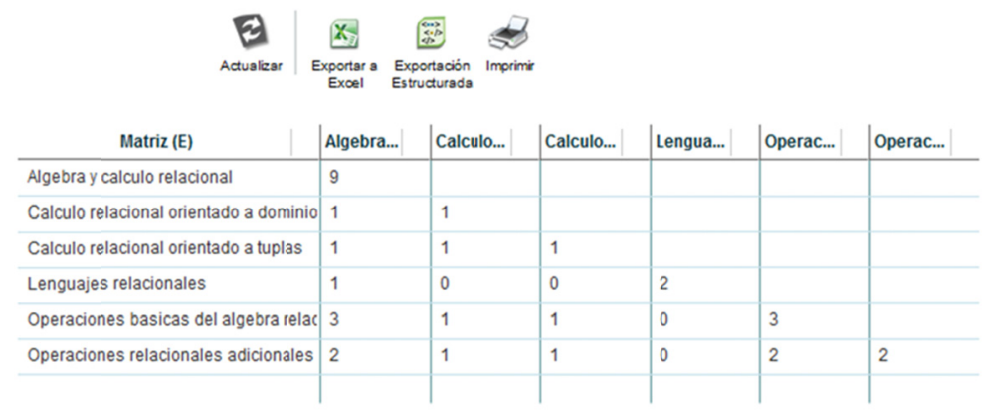

Figura 7 - Ejemplo de matriz triangular.

\subsection{Importación de las matrices en el software Goluca.}

Como resultado de la consulta de la matriz triangular, obtenido en el punto anterior, se ha generado un archivo con determinada información que recogemos e integramos en el programa Goluca mediante la opción de importación de datos. Podemos ver el contenido del fichero generado en WebQDA que importa Goluca en la siguiente figura 8. 


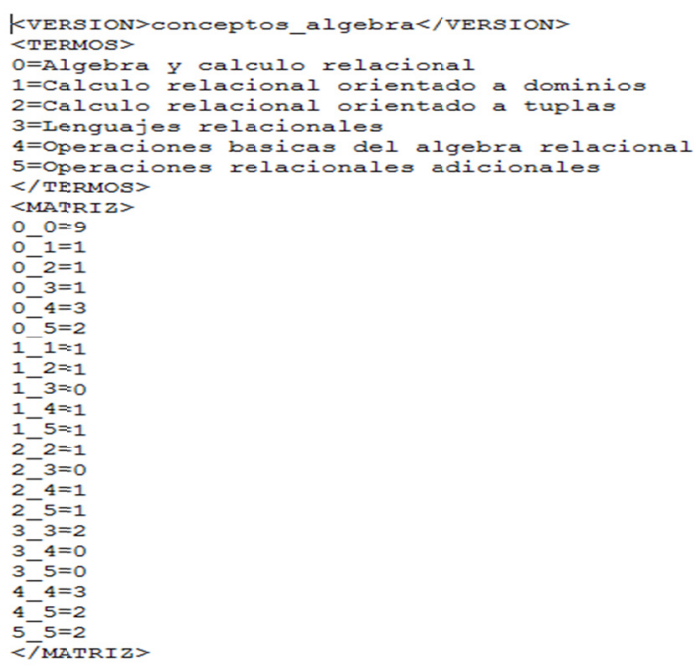

Figura 8 - Datos de la matriz triangular importada a Goluca.

Como podemos observar en la figura anterior. Los datos de la matriz están separados en varios bloques. Todos los bloques comienzan con una etiqueta y finalizan con la misma etiqueta añadiendo como primer carácter el símbolo "/". De todas las etiquetas que hay, las que nos interesan son las tres siguientes:

- $<$ VERSION $>$ nombre</VERSION $>$. Contiene el nombre de la versión del fichero de salida.

- $\quad<$ TERMOS $>0=$ nombreA $1=$ nombreB ...</TERMOS $>$. Contiene los nombres de cada término de la matriz. Cada término tiene un prefijo de un número seguido del símbolo “=” correspondiente a la posición que ocupa ese término dentro de la matriz.

- $<$ MATRIZ $>$ O_O=valor o_1=valor ...</MATRIZ $>$. Contiene los valores de cada celda de la matriz. Teniendo en cuenta lo comentado en el apartado $3.4 \mathrm{y}$ sabiendo que es una matriz simétrica no necesitamos tener representados todos los términos de la matriz, sino solo de los que no se repiten. Es decir, el valor del termino o_1 es igual al valor del término 1_o, por lo que solo aparece una vez uno de ellos.

\subsection{Análisis de la red pathfinder obtenida en el software Goluca}

En el programa Goluca, una vez importada la matriz anterior, utilizando los algoritmos adecuados (Kamada T. and Kawai S., 1989), se procede a representar la Red Pathfinder correspondiente a los valores de la misma y a realizar otros cálculos que nos dan información muy importante sobre la misma red (Godinho V., 2007). De toda la información que tenemos de la Red Pathfinder, la que más nos interesa es la siguiente:

- Índice de complejidad de la red. Este parámetro contiene un valor entre o y 1000 que indica cómo de compleja es la red obtenida, en cuanto a términos 
que tiene y las relaciones entre ellos. A mayor valor mayor complejidad de la red.

- Coherencia de la red. Este parámetro indica si una red puede ser construida fruto del azar, con valores erróneos. Es un valor entre o y 1 donde valores por debajo de o,20 indica posibilidad de redes erróneas o mal construidas, es decir, a mayor valor la red será más coherente.

- Nodos nucleares. Se considera un nodo como nuclear aquel que tiene más de tres enlaces. Por lo que a mayor número de enlaces el nodo será más nuclear o más importante. Teóricamente un concepto nuclear (Luengo R., 2013) es aquel concepto importante que los usuarios tienen más anclados en su mente y que utilizan para organizar y estructurar su red cognitiva.

\subsection{Establecimiento de los Conceptos Nucleares a través de Goluca}

De la distinta información que nos proporciona Goluca, que ya hemos comentado anteriormente, nos interesa fundamentalmente la referida a los Nodos Nucleares, ya que nosotros buscamos los conceptos básicos de Base de Datos con respecto a un amplio número de conceptos.

Como podemos observar en la figura 9 esta figura es un ejemplo con nodos correspondientes al grupo "Modelo entidad Relación"; esta figura representa todos los conceptos analizados en el grupo, y entre los cuales, con color amarillo en un círculo, tenemos los conceptos nucleares. Los más nucleares se diferencian por tener un color amarillo más oscuro con respecto al resto de conceptos nucleares. Los conceptos no nucleares tendrán otro color tal como azul o verde.

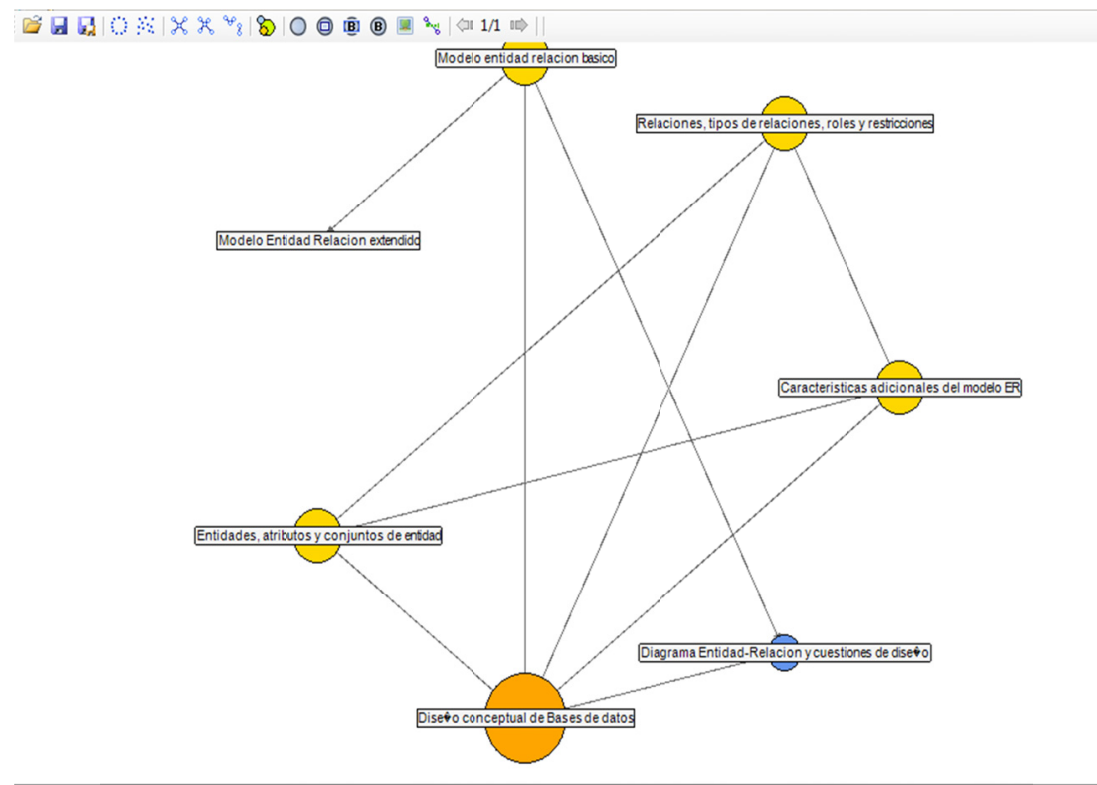

Figura 9 - Representación de los Conceptos Nucleares 


\section{Resultados}

Después de llevar a cabo el proceso reflejado anteriormente, con todos los grupos de que disponíamos, obtuvimos los conceptos nucleares referidos a nuestra investigación sobre el total de los conceptos básicos de Base de Datos. El resultado, en cuanto al número de conceptos por grupo, lo podemos visualizar en la siguiente tabla 1.

Tabla 1 - Número de conceptos no nucleares y nucleares por grupos.

\begin{tabular}{cll}
\hline Grupos & $\begin{array}{l}\text { Conceptos } \\
\text { Totales }\end{array}$ & $\begin{array}{l}\text { Conceptos } \\
\text { Nucleares }\end{array}$ \\
\hline $\begin{array}{c}\text { Introducción y conceptos de los } \\
\text { sistemas de información, base de datos } \\
\text { y sistemas de gestión de base de datos }\end{array}$ & 22 & 19 \\
\hline Modelos de datos & 10 & 10 \\
\hline Modelo Entidad Relación & 7 & 5 \\
\hline Modelo Relacional & 14 & 9 \\
\hline Normalización & 6 & 5 \\
\hline Álgebra y Cálculo Relacional & 6 & 5 \\
\hline SQL (Structured Query Languages) & 12 & 9 \\
\hline Programación y desarrollo de base de \\
datos
\end{tabular}

De los 90 conceptos obtenidos en el análisis cualitativo con el software WebQDA, al proceder a realizar el análisis cuantitativo con el software Goluca, hemos obtenido que sólo 72 conceptos pueden considerarse como los conceptos más importantes. Para nosotros, al ser tales conceptos (nodos) los nodos nucleares, pasarán a ser los conceptos básicos de Base de Datos que estábamos buscando.

En concreto, la relación de los conceptos nucleares que han quedado al final, ordenados por grupos, los podemos visualizar en la siguiente tabla 2:

Tabla 2 - Relación de Conceptos nucleares por grupos.

\begin{tabular}{cl}
\hline Nombre del grupo & Nombre de los conceptos nucleares \\
\hline & Análisis, diseño e implementación de Base de Datos. \\
& Aplicaciones de los sistemas de base de datos. \\
& Arquitecturas de los S.I y SGBD. \\
& Conceptos previos, básicos y generales sobre Base de Datos. \\
& El entorno del SBD y clasificación de los SBD. \\
Introducción y conceptos de los & Independencia de Datos. \\
sistemas de información, base de & Estructura de un SGBD. \\
datos y sistemas de gestión de base & Introducción a las BD y SGBD. \\
& Lenguajes de Bases de Datos. \\
& Objetivos de las bases de datos. \\
& Origen y evolución de las bases de datos. \\
& Perspectiva Histórica. \\
& Porqué usar un SBD. \\
&
\end{tabular}




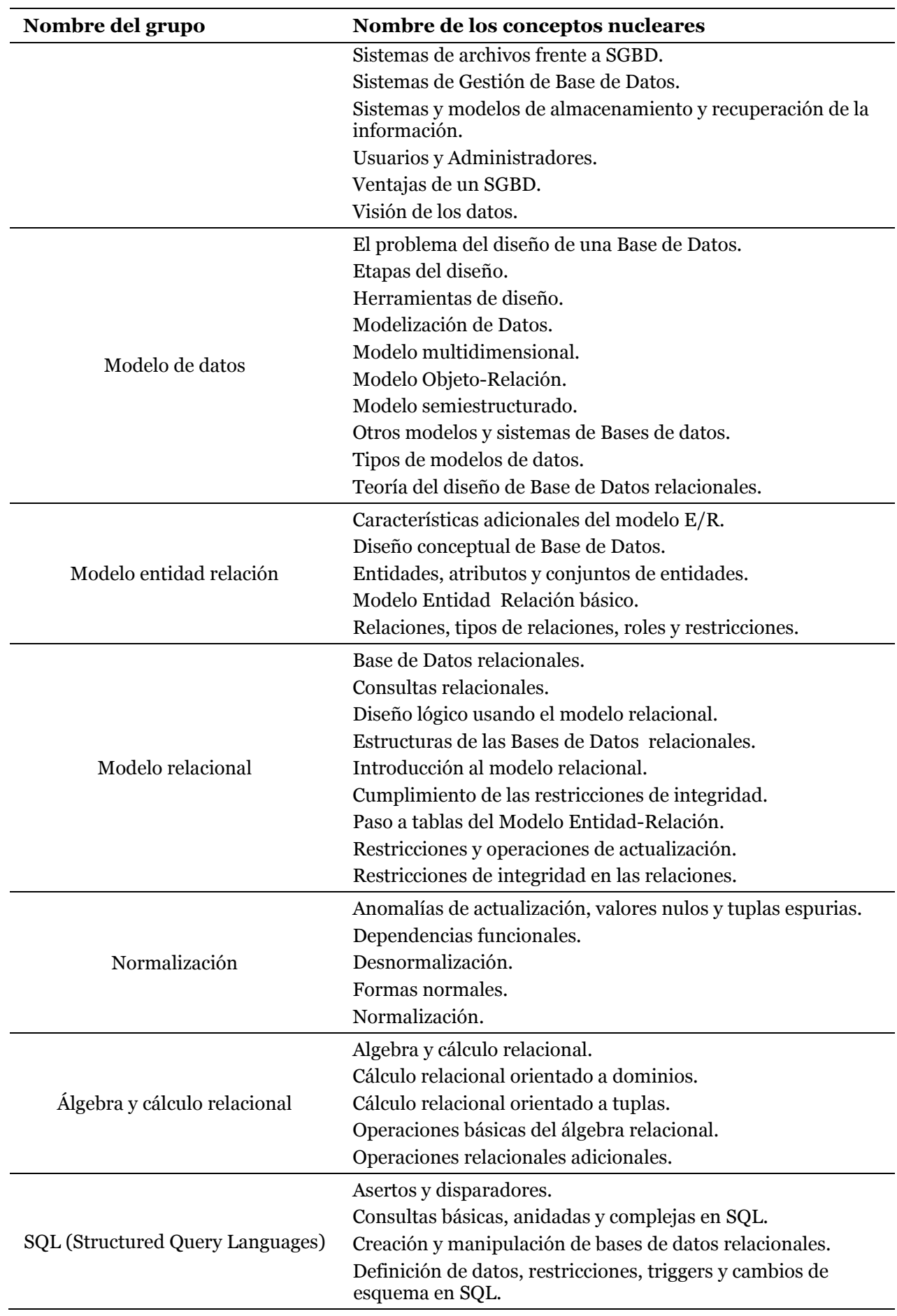




\begin{tabular}{ll}
\hline Nombre del grupo & Nombre de los conceptos nucleares \\
\hline & Lenguajes LDD, LCD y LMD. \\
Modificación de la Base de Datos. \\
Operaciones de agregación. \\
Sentencias INSERT, DELETE y UPDATE. \\
Vistas. \\
\hline Acceso a Base de Datos desde aplicaciones, SQL empotrado y \\
SQL dinámico. \\
Disparadores. \\
Arquitectura de las aplicaciones. \\
Diseño Físico de Base de datos. \\
Procedimientos almacenados en la BD. \\
Elementos de un API de acceso a BD. \\
JDBC. \\
de datos & Otros APIs de acceso a BD. \\
& Programación de Bases de Datos. \\
Transacciones.
\end{tabular}

\section{Conclusiones}

La experiencia de utilizar una investigación mixta, donde se ha utilizado algunos elementos de análisis cualitativo para después finalizar en un entorno cuantitativo, en el que se ha adquirido información precisa sobre nuestros datos, ha sido una experiencia enriquecedora e interesante; y nos ha permitido ajustar algunas cuestiones de ambos software utilizados. Al ser la primera vez que utilizábamos en conjunto ambos programas ha sido necesario el ajuste fundamentalmente focalizado a la transferencia de información entre ambos.

Otra de las cuestiones de las que hemos sacado conclusiones interesantes ha sido la utilización de las RAP cuando el número de nodos de estudio se hace bastante numeroso. Esta técnica mejora bastante con un número de nodos pequeño, pero cuando se hace numeroso el número de nodos, la discriminación de los nodos nucleares se hace más engorrosa. Lo que nos lleva a determinar la necesidad de introducir algún elemento adicional de discriminación para que eso sea fácilmente realizable y que permita graduar más exactamente la diferencia entre ellos.

Por último, y lo que para nosotros ha sido más importante, mediante el uso conjunto de estas dos técnicas de análisis nos ha permitido encontrar los conceptos básicos fundamentales de Base de Datos que pueden ser incluidos en los planes docentes de esta asignatura en los Grados de Informática, adaptados al EEES, en las universidades españolas.

\section{Referencias bibliográficas}

Ausubel D.P., Novak J.D. y Hanesian H. (1978). Psicologia educativa: un punto de vista cognoscitivo. Ed. Trillas. México (trd versión en inglés del 1978). 
Casa L. y Luengo R. (2013). The study of the pupil's cognitive structure: the concept of angle. Eur J Psychol Educ 28:373-398 Springerc (DOI: 10.1007/s10212-012-01194).

Casas L. (2002). El estudio de la estructura cognitiva de los alumnos a traves de redes asociativas Pathfinder. Aplicación y posibilidades en Geometría. Memoria para el Titulo de Doctor . Badajoz, España: Universidad de Extremadura.

Casas L. y Luengo R. (2005). Conceptos nucleares en la construcción del concepto de ángulo. Enseñanza de las Ciencias, n. 23 (2), pag. 201-216.

Casas L. y Luengo R. (2003b). Matemáticas: Representaçao Da estructura Cognitiva de Alunos. Evora, Portugal: Congresso de Neurociencias Cognitivas. Universidade de Évora.

Casas L. y Luengo R. (2004b). Representación del conocimiento y aprendizaje. Teoría de los conceptos nucleares. Revista Española de pedagogía, n. 227, pag. 59-84.

Casas L. y Luengo R. (2004a). Teoría de los Conceptos Nucleares. Aplicación en Didáctica de las Matemáticas. Badajoz: R. Luengo, lineas de investigación en Educación Matemática. Servicio de publicciones FESPM.

Contreras J.A., Arias J., Luengo, R. e Hidalgo V. (2013). Contenidos de las materias de Base de Datos en los Planes de Estudio Universitarios de Grado en Informática en el EEES. $12^{\text {a }}$ Conferencia Iberoamericana en Sistemas, Cibernetica e Informática, Volumne II, págs. 103-108. Orlando, Florida, EEUU.

Contreras J.A., Luengo R., Arias J. y Castillo A. (2013). Análisis Cualitativo de los contenidos de las materias de Base de Datos en los Planes de Estudio Universitarios de Grado en Informática en el EEES. $8^{\text {a }}$ Conferencia Ibérica de Sistemas y Tecnologías de la Información, Volumne I, págs. 395-399. Lisboa, Portugal.

Edelman G. (1992). Bright air, brilliant fire. On the matter of the mind. New York: Basic Books .

Fenker R.M. (1975). The organization of conceptual materials: A methodology for meassuring ideal and actual cognitive structures. Instructional Science , 4, 33-57.

Godinho V. (2007). Implementación del Software Goluca y aplicación al cambio de redes conceptuales. Memoria de Diploma de Estudios Avanzados . Badajoz, España: Universidad de Extremadura.

Jonassen D.H. (1990). Semantic network elicitation: tools for structuring hypertext. Hypertext: State of the Art. Oxford: Intellect .

Kamada T. and Kawai S. (1989). An algorithm for drawing general undirected graphs. Information Processing Letters , 31, 7-15.

Luengo R. (2013). La teoría de los conceptos nucleares y su aplicación de la investigación en la didáctica de las Matemáticas. Revista Iberoamericana de Educación Matemática (34), 9-36. 
Luengo R., Casas L., Mendoza M. y Arias J. (2011). Posibilities of "Nuclear Concepts Theory" on Educacional Research, a Review. Florencia, 16-17 de Junio de 2011: International Conference The Future of Education.

Piaget J. (1978). La evolución intelectual entre la adolescencia y la edad adulta. J Delval comp. Lecturas de Psicología del niño, V 2.

Preece P. (1976). Mapping cognitive structure: A comparison of methods. Journal of Educational Psychology , 68, 1-8.

Rumelhart D.E. (1980). Schemata: The building block of cognition. R.J. Spiro, B.C. Bruce y W. Brewer.Theoretical issues in reading comprehesion. Hillsdale, NJ: Erbaum .

Schvaneveldt R.W. (1990). Pathfinder Associative Networks: Studies in Knowlegde Organization. Norwood, New Jersey, EEUU: ABLEX Publishing Corporation.

Shavelson R. (1972). Some aspects of the correspondence between content structure and cognitive structure in physics instruction. Journal of Educational Psychology , $63,225-234$. 